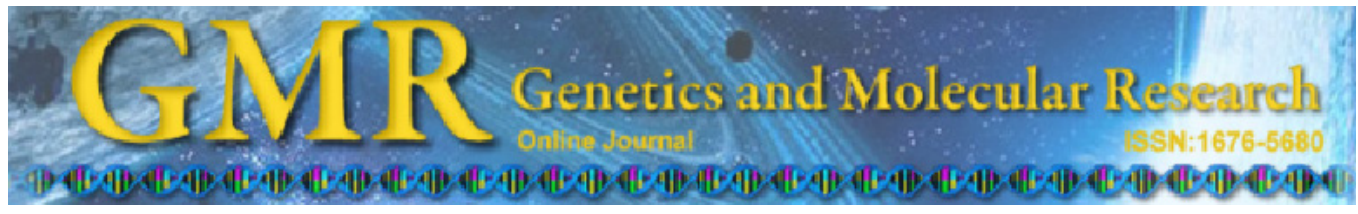

\title{
Phylogenetic relationships of the Hucul horse from Romania inferred from mitochondrial D-loop variation
}

\author{
S.E. Georgescu, M.A. Manea, A. Dudu and M. Costache \\ Department of Biochemistry and Molecular Biology, Faculty of Biology, \\ University of Bucharest, Bucharest, Romania \\ Corresponding authors: S.E. Georgescu / M. Costache \\ E-mail: georgescu_se@yahoo.com / marietacostache@yahoo.com
}

Genet. Mol. Res. 10 (4): 4104-4113 (2011)

Received March 25, 2011

Accepted August 8, 2011

Published October 31, 2011

DOI http://dx.doi.org/10.4238/2011.October.31.7

\begin{abstract}
The existence of the Hucul horse on Romanian territory has been documented from the very distant past; today Hucul is a unique breed that is part of the FAO Program for the Preservation of Animal Genetic Resources. We compared Hucul with several primitive European and Asiatic breeds in order to elucidate the origin of these horses. We analyzed a 683-bp mitochondrial DNA (mtDNA) D-loop fragment in a population of Hucul horses and compared the polymorphic sites with sequences from other primitive breeds, including Exmoor, Icelandic Pony, Sorraia, Przewalski Horse, Mongolian Wild Horse, Konik, and Shetland Pony, as well as with Arabian, Akhal Teke and Caspian Pony. The sequences were truncated to $247 \mathrm{bp}$ to accommodate short sequence data for the other species. Eighty horses were analyzed; 35 polymorphic sites representing 33 haplotypes were observed. The mean percentage of polymorphic sites was $14.2 \%$ for this mtDNA fragment. A neighbor-joining phylogenetic tree was constructed based on Kimura two-parameter distances and the Network 3.111 software was used for phylogenetic analysis. The Hucul horse was classified separately from all other primitive breeds. It is possible that the Hucul horse is not part of the pony class, as it segregated apart from all primitive pony breeds.
\end{abstract}


We found multiple origins in the maternal lineage of domestic horse breeds and demonstrated the uniqueness of the Hucul breed; its origins remain unclear.

Key words: Hucul horse; Primitive; Mitochondrial DNA; Phylogeny

\section{INTRODUCTION}

Currently, it is thought that at the end of the last glacial era wild horses on the American continent disappeared, while in Eurasia they managed to survive until a few centuries back. In this geographical area, it is believed that various subspecies of wild horses were in existence, including Tarpan (Equus ferus ferus) in the European area and the Przewalski horse (Equus ferus przewalskii) in the Asian area (Kavar and Dove, 2008). The latter was saved from extinction due to lucky circumstances, and thus, the population of Przewalski horses that live free nowadays are descendents of a much reduced number of individuals from zoological gardens.

Nevertheless, it is believed that the Tarpan horse still has several descendents among current horse breeds. Therefore, the primitive Polish Konik horse seems to be a direct descendent of the Tarpan horse. This horse has a great number of primitive traits which bring it closer to the Tarpan horse. Other possible descendents of the Tarpan are the Sorraia horses from Portugal. These are considered to be the remainders of an old population of wild horses that have survived, until the present time, in the lower areas of the Sorraia River in Portugal. This population was discovered only in the 1920 s, and currently there are only about 200 individuals (Lopes et al., 2005). If the area in which the Tarpan lived several thousand years ago was far more extended than merely the East European region, these Sorraia horses could then represent the last subpopulation that could have inherited the characteristics of the Tarpan.

Regarding the horses of the Hucul breed, their origin is controversial. The Hucul could be considered a direct descendent of the Tarpan horse. The name of these horses originates from the ethnic group that originally bred them, the Hutsul group. The Hutsul ethnic group belonged to the Slavic populations who lived in the mountainous areas near the sources of the Siret and Ceremus rivers, and who spoke a Ukrainian dialect. The morphological characteristics of the Hucul horses make them similar to the Tarpan. These are small horses, perfectly adjusted to mountainous areas, possessing great endurance in the wilderness; they can easily find their food and are extremely resistant to disease. As an adaptation trait, to the mountainous areas in which they live, these horses have very resistant hooves and do not require horseshoes. In the 19th century, the first Hucul stud farm was established in Radauti, present-day Romania, and five bloodlines were established and named after the foundation stallions: Goral, Hroby, Ouşor, Pietrousu and Prislop. Currently, Hucul is bred on stud farms in Slovakia, Romania and Poland.

In order to investigate the origins of this particular horse breed we have resorted to molecular methods that comprised the comparative analysis of the mitochondrial D-loop region in various primitive breeds spread around Eurasia and in Hucul horses originating in Romania.

Due to maternal inheritance, high copy number in the cells, the lack of recombination and their fast molecular evolution, mtDNA is widely used in molecular phylogeny studies. If no mutation is apparent, the filiations will have the same haplotype as the ascendant of the maternal line. The mutations that appear occasionally are retained at the level of the maternal lines 
and their study helps us track their evolution in time. A relevant percentage of the studies undertaken with the help of mtDNA at the level of horse breeds and populations have focused on the analysis of the nucleotide sequence of the mitochondrial D-loop region (Kavar et al., 1999, Kim et al., 1999, Vila et al., 2001, Lopes et al., 2005, McGahern et al., 2006, Aberle et al., 2007). This is represented by a sequence of about $1200 \mathrm{bp}$, flanked by the encoding regions of tRNAs for proline and phenylalanine. The D-loop region contains two variable portions (HVR1 and HVR2), four conserved nucleotide blocks (CSB 1-4) and a variable area containing a repeated motif of 8 bp with the sequence GTGCACCT (Xu and Arnason, 1994; Ishida et al., 1994).

The aim of this study was to compare the Hucul breed with various primitive horse breeds from Europe and Asia in order to investigate their origins. The comparative analysis was performed at the molecular level by analyzing a fragment from the mitochondrial Dloop region.

\section{MATERIAL AND METHODS}

\section{DNA extraction}

mtDNA D-loop sequences were determined for 29 Hucul horses from Lucina Stud, Romania. DNA was extracted from blood samples preserved in EDTA. The isolation of genomic DNA from fresh blood was performed with the Wizard Genomic DNA Extraction kit (Promega) according to manufacturer instructions.

\section{DNA amplification and sequencing}

PCR was done using a GeneAmp 9700 PCR System (AppliedBiosystems). The reactions were carried out in a $25-\mu \mathrm{L}$ final volume containing PCR buffer, $1.5 \mathrm{mM} \mathrm{MgCl}, 200$ $\mu \mathrm{M}$ dNTPs, and $0.5 \mu \mathrm{M}$ of each primer (F: 5'-caacacccaaagctgaaattcta-3'; R: 5'-gctaccatg gactgaataacacc-3'), 0.5 U AmpliTaq Gold DNA Polymerase, DNA and nuclease-free water. PCR was performed using 40 cycles of denaturation at $95^{\circ} \mathrm{C}(30 \mathrm{~s})$, annealing at $57^{\circ} \mathrm{C}(30$ s) and extension at $72^{\circ} \mathrm{C}(60 \mathrm{~s})$. The first denaturation step was 10 min at $95^{\circ} \mathrm{C}$ and the last extension was $10 \mathrm{~min}$ at $72^{\circ} \mathrm{C}$. The primers were designed to amplify a fragment of $683 \mathrm{bp}$ from the tRNA ${ }^{\text {Pro }}$ gene and D-loop region (from 15,430 to $16,112 \mathrm{bp}$ ). Our nucleotide position (np) numbering followed that of Xu and Arnason (1994).

PCR products were detected by electrophoresis on a $2 \%$ agarose gel stained with ethidium bromide and then purified with the Wizard PCR Preps DNA Purification System kit (Promega). The amplified fragments were sequenced with an ABI Prism 3130 Genetic Analyzer, using the ABI Prism ${ }^{\circledR}$ BigDye Terminator Cycle Sequencing Reaction kit (Applied Biosytems). The sequences were processed using DNA Sequencing Analysis 5.1 software (Applied Biosytems), and the nucleotide sequences were aligned with the BioEdit program (Hall, 1999 ) and refined manually. Partial sequences for the tRNA ${ }^{\text {Pro }}$ gene and D-loop region were deposited in GenBank under the following accession numbers: GU339387 and GU339388.

\section{Sequence alignment and molecular phylogenetic analysis}

The D-loop sequences obtained were edited by the BioEdit program. Sequences were 
truncated to nps 15494-15740 to accommodate short sequences already published. The sequences were aligned using the ClustalX 2.0.9 software (Larkin et al., 2007) with 10-gap opening penalty, 0.10 -gap extension penalty and the rest of the settings as default.

For a complex phylogenetic evaluation, we included mtDNA control region sequences, which were available from GenBank or from publications. Equus asinus was selected as the outgroup. The GenBank accession numbers and references for the published sequences are presented in Table 1.

Table 1. GenBank accession numbers and references for published sequences.

\begin{tabular}{|c|c|c|c|c|}
\hline & Abbreviation & Accession number & Breed & Reference \\
\hline 1 & AKT1 & DQ327964 & Akhal Teke & McGahern et al., 2006 \\
\hline 2 & AKT2 & DQ327962 & & \\
\hline 3 & AKT3 & DQ327960 & & \\
\hline 4 & AKT4 & DQ327959 & & \\
\hline 5 & AKT5 & DQ327958 & & \\
\hline 6 & AKT6 & DQ327957 & & \\
\hline 7 & AKT7 & DQ327956 & & \\
\hline 8 & AKT8 & DQ327953 & & \\
\hline 9 & AKT9 & DQ327952 & & \\
\hline 10 & AKT10 & DQ327950 & & \\
\hline 11 & KON1 & AJ413731 & Konik & Jansen et al., 2002 \\
\hline 12 & KON2 & AJ413732 & & \\
\hline 13 & KON3 & AJ413733 & & \\
\hline 14 & KON4 & AJ413734 & & \\
\hline 15 & MWH1 & AJ413830 & Mongolian wild horse & Jansen et al., 2002 \\
\hline 16 & MWH2 & AJ413832 & & \\
\hline 17 & NOR1 & AJ413825 & Noriker & Jansen et al., 2002 \\
\hline 18 & NOR2 & AJ413826 & & \\
\hline 19 & NOR3 & AJ413828 & & \\
\hline 20 & CAS1 & AJ413724 & Caspian pony & Jansen et al., 2002 \\
\hline 21 & CAS2 & AJ413726 & & \\
\hline 22 & CAS3 & AJ413728 & & \\
\hline 23 & CAS4 & AJ413730 & & \\
\hline 24 & EXM1 & AJ413682 & Exmoor & Jansen et al., 2002 \\
\hline 25 & EXM2 & AJ413684 & & \\
\hline 26 & EXM3 & AJ413691 & & \\
\hline 27 & EXM4 & AJ413692 & & \\
\hline 28 & FRI1 & AJ413705 & Friesian & Jansen et al., 2002 \\
\hline 29 & FRI2 & AJ413706 & & \\
\hline 30 & ICP1 & AJ413717 & Icelandic pony & Jansen et al., 2002 \\
\hline 31 & $\mathrm{ICP} 2$ & AJ413718 & & \\
\hline 32 & ICP3 & AJ413720 & & \\
\hline 33 & ICP4 & AJ413721 & & \\
\hline 34 & ICP5 & AJ413722 & & \\
\hline 35 & ICP6 & AJ413723 & & \\
\hline 36 & NFJ1 & AJ413694 & Norwegian Fjord & Jansen et al., 2002 \\
\hline 37 & NFJ2 & AJ413695 & & \\
\hline 38 & NFJ3 & AJ413699 & & \\
\hline 39 & NFJ4 & AJ413701 & & \\
\hline 40 & NFJ5 & AJ413702 & & \\
\hline 41 & NFJ6 & AJ413704 & & \\
\hline 42 & SHP1 & AJ413919 & Shetland pony & Jansen et al., 2002 \\
\hline 43 & SHP2 & AJ413920 & & \\
\hline 44 & SCH1 & AJ413866 & Scottish Highland & Jansen et al., 2002 \\
\hline 45 & $\mathrm{SCH} 2$ & AJ413867 & & \\
\hline 46 & $\mathrm{SCH} 3$ & AJ413869 & & \\
\hline 47 & $\mathrm{SCH} 4$ & AJ413871 & & \\
\hline 48 & SOR1 & AJ413901 & Sorraia & Jansen et al., 2002 \\
\hline 49 & SOR2 & AJ413904 & & \\
\hline 50 & PZW & AF014409 & Przewalski horse & Kim et al., 1999 \\
\hline 51 & ARA & AJ413648 & Arabian & Jansen et al., 2002 \\
\hline 52 & & X97337 & Equus asinus & Xu et al., 1996 \\
\hline
\end{tabular}


A neighbor-joining (NJ) phylogenetic tree was constructed on the basis of Kimura 2-parameter distances (Kimura, 1980). Bootstrap analyses (1000 replications) were used to assess the confidence of each node. Phylogenetic analyses were conducted using the MEGA software version 4.0 (Tamura et al., 2007).

NETWORK 3.111 software (http://fluxus-engineering.com) was also used for phylogenetic analysis. Network building algorithms begin with raw sequences, rather than distance data, and generate their own genetic distances from these data. This represents another way to approach evolutionary relationships, because it allows reticulation and presumes branching evolution (Bandelt et al., 1999; Posada and Crandall, 2001).

\section{RESULTS}

In this study, we determined partial sequences for the mitochondrial D-loop region in the Hucul horse breed from Romania. For a complex overview of the phylogenetic information content, the D-loop sequence was aligned and compared with 52 similar GenBank sequences from other primitive horse breeds.

A fragment of $247 \mathrm{bp}$ was aligned, 35 sites were variable (34 transitions and 1 transversion) and represented $14.17 \%$ of the total number. Considering only different haplotypes per breeds and ignoring shared haplotypes, there was a total of 33 haplotypes in all horse populations. Transitions were the most common substitutions detected. The polymorphic sites observed per breed are shown in Table 2, and the results obtained in terms of nucleotide variation and haplotypes are shown in Figure 1.

As a result of the analyses of the 15 breeds, a great number of haplotypes were observed (Figure 1). In general, most breeds considered showed distinct haplotypes, with some exceptions. Thus, in the Akhal Teke breed there were 9 different haplotypes, some of which were common with those encountered in other breeds which are apparently not kindred and which are situated in different geographical areas. For instance, the H2 haplotype was shared between the Akhal Teke, Friesian and Caspian pony breeds, and the H3 haplotype was common for the Akhal Teke, Noriker and Scottish Highland, while the H11 haplotype was common for the Akhal Teke, Hucul and Shetland pony. In addition, the Akhal Teke breeds shared the H19 and H21 haplotypes with the Icelandic pony, Norwegian Fjord, Scottish Highland and Konik breeds.

At the same time, the Hucul horses showed four haplotypes: H11, H22, H25 and H28. In fact, it is very interesting that the haplotype H28 found in the Romanian Hucul did not occur in the other breeds analyzed. Certain unique haplotypes were also present in the case of the Sorraia breed, a possible descendent of the Tarpan breed for the Western European region.

Also, the Mongolian wild horse and Przewalski horse showed distinct haplotypes (H14 and H15), clearly different from other breeds. Another breed with shared haplotypes was the Icelandic pony. Therefore, haplotypes H1, H4, H18, H19 and H24 were shared among Norwegian Fjord, Caspian Pony, Akhal Teke, Scottish Highland, Exmoor and Icelandic pony.

In order to construct the phylogenetic tree and network, we used the same 15 primitive breeds. It was appealing to analyze the position of the Hucul horse in relation to these ancient breeds. In this study, phylogenetic analysis was based on D-loop region partial sequences. The NJ phylogenetic tree (Figure 2) was constructed with Equus asinus (GenBank Accession No. X97337) as the outgroup. Support for individual branch of NJ phylogenetic tree was assessed 
by bootstrap percentages (BP) computed after 1000 replicates of the closest stepwise addition option. Concurrently, we constructed the rooted evolutionary network (Figure 3) using 15 horse breeds and Equus asinus as the outgroup.

\begin{tabular}{|c|c|c|c|c|c|}
\hline Breed name and abbreviation & No. of individuals & Polymorphic sites & Haplotypes & Transitions & Transversions \\
\hline Akhal Teke (AKT) & 10 & 20 & 9 & 20 & - \\
\hline Konik (KON) & 4 & 11 & 4 & 11 & - \\
\hline Mongolian wild horse (MWH) & 2 & 8 & 2 & 7 & 1 \\
\hline Noriker (NOR) & 3 & 14 & 3 & 14 & - \\
\hline Caspian pony (CAS) & 4 & 10 & 4 & 10 & - \\
\hline Exmoor (EXM) & 4 & 9 & 3 & 9 & - \\
\hline Friesian (FRI) & 2 & 10 & 2 & 10 & - \\
\hline Icelandic pony (ICP) & 6 & 19 & 6 & 19 & - \\
\hline Norwegian Fjord (NFJ) & 6 & 20 & 5 & 20 & - \\
\hline Shetland pony (SHP) & 2 & 11 & 2 & 11 & - \\
\hline Scottish Highland (SCH) & 4 & 12 & 4 & 12 & - \\
\hline Sorraia (SOR) & 2 & 9 & 2 & 9 & - \\
\hline Przewalski horse (PZW) & 1 & 7 & 1 & 7 & - \\
\hline Arabian (ARA) & 1 & 5 & 1 & 5 & - \\
\hline Hucul (HUC) & 29 & 11 & 4 & 11 & - \\
\hline
\end{tabular}

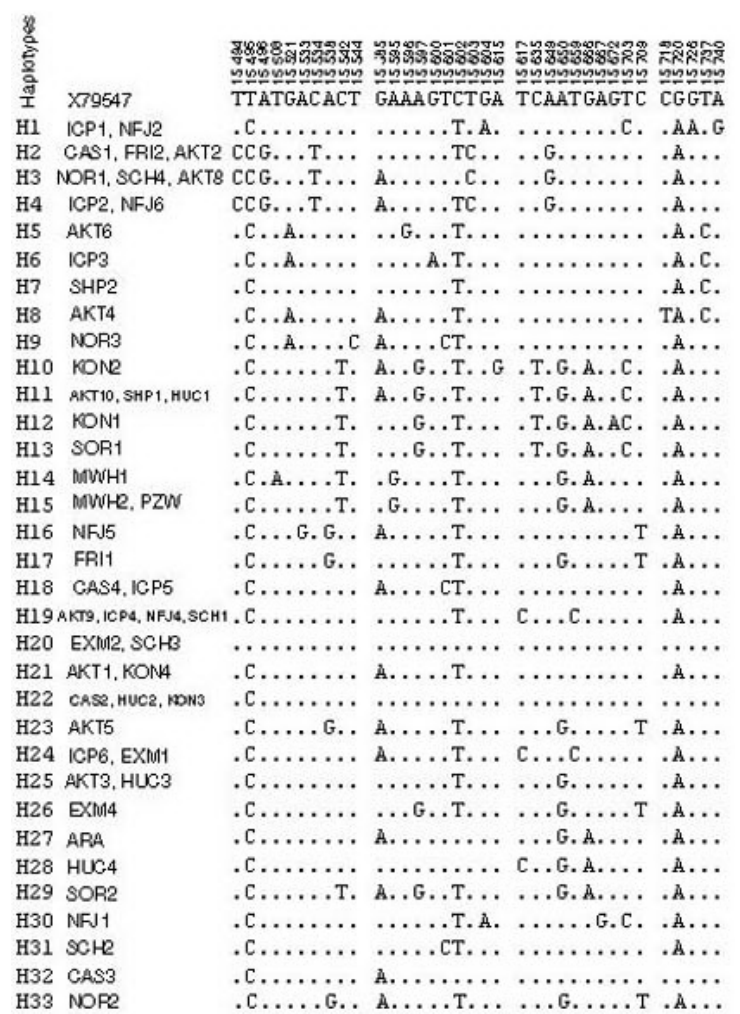

Figure 1. Haplotypes and variable sites in D-loop region (between nucleotides 15494 and 15740) in 15 horse breeds and the reference sequence (GenBank X79547; Xu and Arnason, 1994). The numbers represent the position occupied in the D-loop region, respectively. Identical sites are indicated by the symbol ".". 


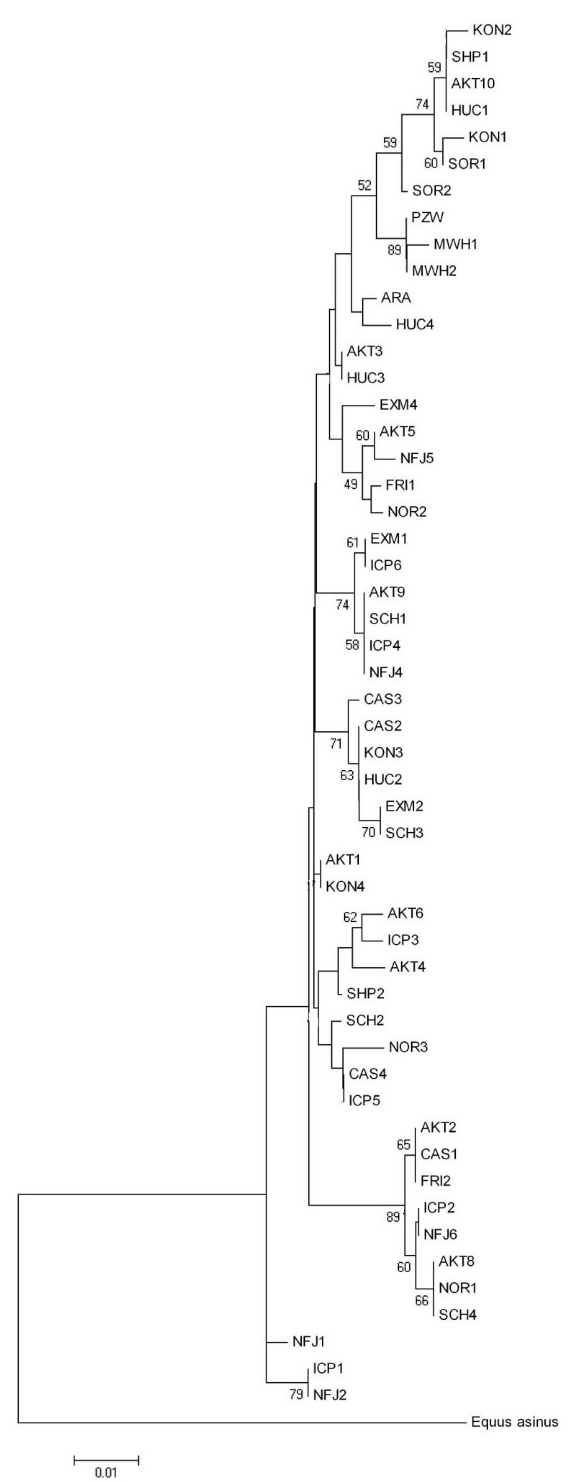

Figure 2. Neighbor-joining tree of 33 different haplotypes, including 15 horse breeds and the sequence of Equus asinus as the outgroup. Figures on the internodes are bootstrap probabilities based on 1000 replications.

The Przewalski and Mongolian wild horse were situated in the same group both at the level of the phylogenetic tree (Figure 2), as well as the level of the network (Figure 3). The individuals belonging to these two populations had two distinctive haplotypes (H14 and H15), which did not exist in the other breeds.

The separation of clusters in the network may represent additional proof of the evolution in particular conditions of these ancient horse breeds. Thus, Sorraia horses originated from a group of 7 mares and 4 stallions obtained by R. d'Andrade near Coruche, Portugal 
around 1930, after he had seen a phenotypically identical wild population there in 1920 (Jansen et al., 2002). Concurrently, just as in the case of the phylogenetic tree, the Przewalski and Mongolian wild horse breeds formed, as expected, a totally separate cluster.

The analysis of the phylogenetic network led to observations regarding the clear separation of several breed groups, including the Sorraia, Przewalski, Mongolian wild horse and Hucul breeds. At the same time, we can observe a major group (Cluster1) that includes most of the breeds analyzed (Figure 3).

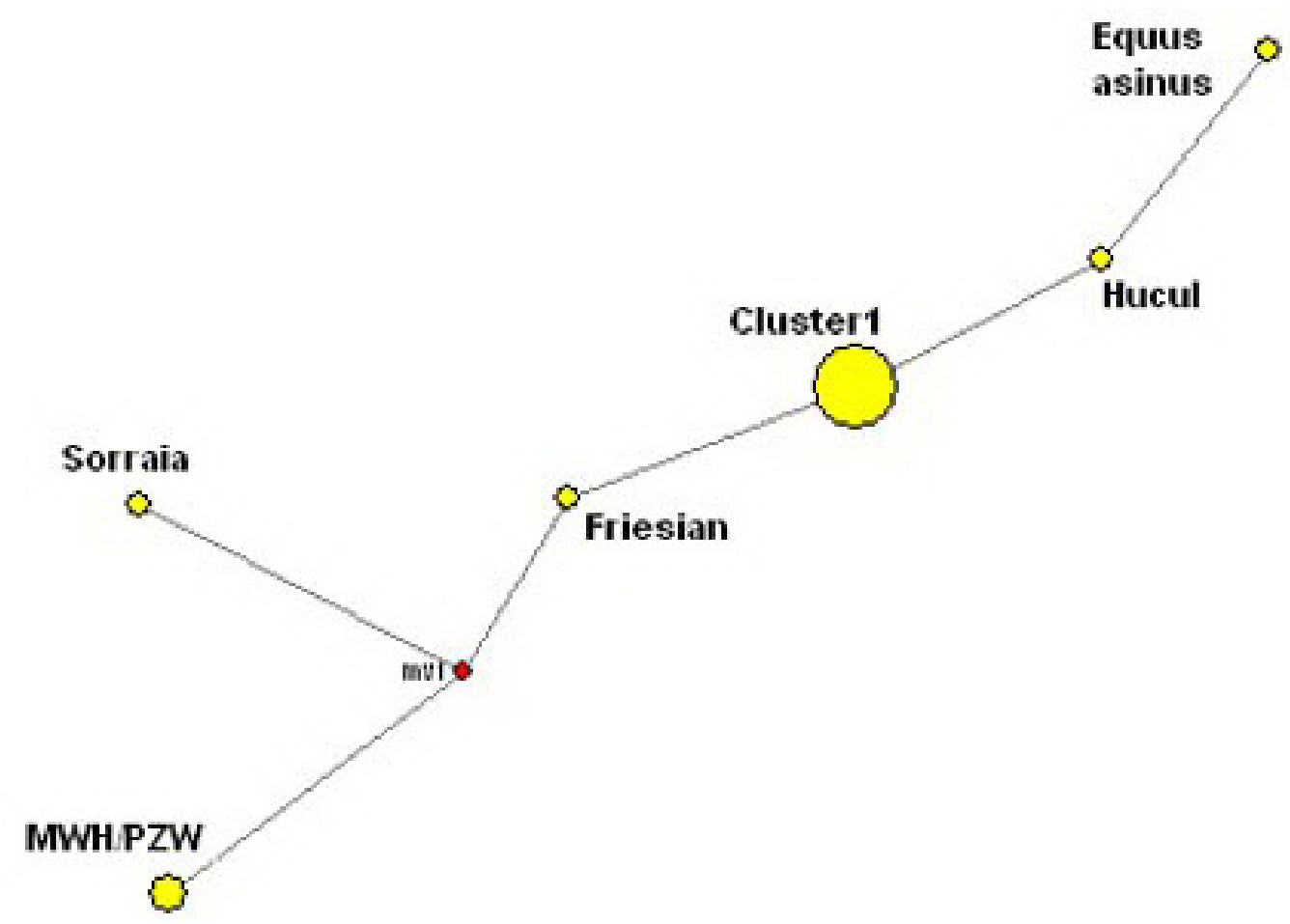

Figure 3. Phylogenetic network of D-loop sequences from 80 horses. Circles are proportional to the number of horses they represent. The node "Equus asinus" is the root of the network according to equid outgroups. Circle names and theirs representation: Equus asinus - Equus asinus, Hucul - Hucul, Sorraia - Sorraia, MWH/PZW Mongolian wild horse and Przewalski horse, Friesian - Friesian, Cluster1 - Exmoor, Icelandic pony, Konik, Noriker, Norwegian Fjord, Arabian, Scottish Highland, Shetland pony, Akhal Teke and Caspian pony.

\section{DISCUSSION}

Until now, from various breeds all over the world, over 100 distinct mtDNA haplotypes have been obtained (Kavar and Dovc, 2008). Among the first breeds investigated were Lipizzaner (Kavar et al., 1999) and Arabian (Bowling et al., 2000). In native or primitive breeds, or in a breed with a small population size, the extent of mtDNA variation is smaller. In a previous study, seven distinct haplotypes were detected by sequence analysis of 20 Exmoor ponies while the variation in the Sorraia breed was limited to two distinct haplotypes (Kavar and Dovc, 2008). 
However, our analyses of 15 primitive horse breeds revealed a high genetic variability. According to Cieslak et al. (2010), the reasons for the presence of such a large amount of genetic variation in the domestic horse are due to a combination of factors: multiple origins, a large number of female founders and a large-scale introgression of local lineages into the domestic stock.

The presence of 33 mtDNA haplotypes with a total of $14.17 \%$ polymorphic sites in a 247-bp fragment in 80 horses belonging to 15 different primitive horse breeds indicates a broad genetic base of maternal lines. This high level of variability is also highlighted in earlier horse mtDNA studies. For example, Yang et al. (2002) found 6\% polymorphic sites defining 17 haplotypes in 65 Cheju horses. Also, Aberle et al. (2007) found 10\% 24 haplotypes and $10 \%$ polymorphic sites in 27 individuals belonging to nine heavy horse breeds. Cieslak et al. (2010) concluded that the large diversity of horse mtDNA lineages is not a product of breeding but represents, in fact, ancestral variability.

In the neighbor-joining tree, just three Hucul haplotypes ( $\mathrm{H} 1, \mathrm{H} 3$ and $\mathrm{H} 4)$ were grouped in the same robust cluster while $\mathrm{H} 2$ haplotype was in a separate position (Figure 2). Phylogenetic network shows that 33 haplotypes were grouped into 5 different clusters. The main cluster (Cluster1) contained 23 haplotypes belonging to 10 distinct breeds (Figure 3). The Hucul horse was totally separate from the other breeds, which suggests that it is a distinct population. A separate distribution was found for Sorraia and Friesian, but also for two Asian wild breeds (Mongolian wild horse and Przewalski horse). Another important observation regards the Konik breed, which is considered a descendent of the Tarpan horses and similar to the Hucul, but which was separate from the latter and was located in the main cluster of the phylogenetic network, together with other breeds such as the Icelandic pony, Exmoor, shetland Pony, Akhal Teke or Caspian Pony.

The Hucul horse is considered the most valuable equine material in the area of the Carpathian Mountains and it is the only autochthonous primitive breed surviving over the centuries in this geographic region. The origins of this mountain horse are still under question. On the one hand, the Hucul are thought to be the result of cross-breeding between Tarpan horses and the Mongol domestic horses brought to the Carpathian Mountains during the migration of peoples from the Central Asian region. On the other hand, it is possible that they are the direct descendents of the Tarpan horses, in fact, representing a subpopulation of Tarpan horses that adapted to the conditions in mountainous areas. It is considered that these horses have in fact more common characteristics with the Tarpan horses than even the Konik horses, since the cross-breeding phenomenon with other breeds is nonexistent in the case of the Hucul breed.

The present study confirmed once again the multiple origins in the maternal lineage of domestic horse breeds observed by other investigators (Kim et al., 1999; Bowling et al., 2000; Yang et al., 2002; Aberle et al., 2007; Cieslak et al., 2010). Therefore, in what concerns the origins of the Hucul horse, they are still controversial, as this study only emphasized the uniqueness of this breed. If we were to broaden the scope of the molecular analyses to other lines of Hucul horses from countries such as Poland or Slovakia, this could contribute to the discernment of the affiliation of this breed with a certain group of horses.

Analysis of the mitochondrial D-loop region suggests that the Hucul horse is not part of the pony class, because it segregates completely from all primitive pony breeds. Therefore, the height and morphological characteristics that encouraged its inclusion in this category may have simply been the result of the adaptation to climatic conditions in the mountainous areas. 


\section{REFERENCES}

Aberle KS, Hamann H, Drogemuller C and Distl O (2007). Phylogenetic relationships of German heavy draught horse breeds inferred from mitochondrial DNA D-loop variation. J. Anim. Breed. Genet. 124: 94-100.

Bandelt HJ, Forster P and Rohl A (1999). Median-joining networks for inferring intraspecific phylogenies. Mol. Biol. Evol. 16: 37-48.

Bowling AT, Del VA and Bowling M (2000). A pedigree-based study of mitochondrial D-loop DNA sequence variation among Arabian horses. Anim. Genet. 31: 1-7.

Cieslak M, Pruvost M, Benecke N, Hofreiter M, et al. (2010). Origin and history of mitochondrial DNA lineages in domestic horses. PLoS One 5: e15311.

Hall TA (1999). BioEdit: a user-friendly biological sequence alignment editor and analysis program for Windows 95/98/ NT. Nucleic Acids Symp. 41: 95-98.

Ishida N, Hasegawa T, Takeda K, Sakagami M, et al. (1994). Polymorphic sequence in the D-loop region of equine mitochondrial DNA. Anim. Genet. 25: 215-221.

Jansen T, Forster P, Levine MA, Oelke H, et al. (2002). Mitochondrial DNA and the origins of the domestic horse. Proc. Natl. Acad. Sci. U. S. A. 99: 10905-10910.

Kavar T and Dovc P (2008). Domestication of the horse: Genetic relationships between domestic and wild horses. Livest. Sci. 116: 1-14.

Kavar T, Habe F, Brem G and Dove P (1999). Mitochondrial D-loop sequence variation among the 16 maternal lines of the Lipizzan horse breed. Anim. Genet. 30: 423-430.

Kim KI, Yang YH, Lee SS, Park C, et al. (1999). Phylogenetic relationships of Cheju horses to other horse breeds as determined by mtDNA D-loop sequence polymorphism. Anim. Genet. 30: 102-108.

Kimura M (1980). A simple method for estimating evolutionary rates of base substitutions through comparative studies of nucleotide sequences. J. Mol. Evol. 16: 111-120.

Larkin MA, Blackshields G, Brown NP, Chenna R, et al. (2007). Clustal W and Clustal X version 2.0. Bioinformatics 23: 2947-2948.

Lopes MS, Mendonça D, Cymbron T, Valera M, et al. (2005). The Lusitano horse maternal lineage based on mitochondrial D-loop sequence variation. Anim. Genet. 36: 196-202.

McGahern AM, Edwards CJ, Bower MA, Heffernan A, et al. (2006). Mitochondrial DNA sequence diversity in extant Irish horse populations and in ancient horses. Anim. Genet. 37: 498-502.

Posada D and Crandall KA (2001). Intraspecific gene genealogies: trees grafting into networks. Trends Ecol. Evol. 16: $37-45$.

Tamura K, Dudley J, Nei M and Kumar S (2007). MEGA4: molecular evolutionary genetics analysis (MEGA) software version 4.0. Mol. Biol. Evol. 24: 1596-1599.

Vila C, Leonard JA, Gotherstrom A, Marklund S, et al. (2001). Widespread origins of domestic horse lineages. Science 291: 474-477.

Xu X and Arnason U (1994). The complete mitochondrial DNA sequence of the horse, Equus caballus: extensive heteroplasmy of the control region. Gene 148: 357-362.

Xu X, Gullberg A and Arnason U (1996). The complete mitochondrial DNA (mtDNA) of the donkey and mtDNA comparisons among four closely related mammalian species-pairs. J. Mol. Evol. 43: 438-446.

Yang YH, Kim KI, Cothran EG and Flannery AR (2002). Genetic diversity of Cheju horses (Equus caballus) determined by using mitochondrial DNA D-loop polymorphism. Biochem. Genet. 40: 175-186. 